\title{
Quantum Stochastic Synchronization
}

\author{
Igor Goychuk, ${ }^{1}$ Jesús Casado-Pascual, ${ }^{2}$ Manuel Morillo, ${ }^{2}$ Jörg Lehmann, ${ }^{3}$ and Peter Hänggi ${ }^{1}$ \\ ${ }^{1}$ Institut für Physik, Universität Augsburg, Universitätsstrasse 1, D-86135 Augsburg, Germany \\ ${ }^{2}$ Física Teórica, Universidad de Sevilla, Apartado de Correos 1065, Sevilla 41080, Spain \\ ${ }^{3}$ Departement für Physik und Astronomie, Universität Basel, Klingelbergstrasse 82, CH-4056 Basel, Switzerland
}

(Received 14 March 2006; published 21 November 2006)

\begin{abstract}
We study, within the spin-boson dynamics, the synchronization of a quantum tunneling system with an external, time-periodic driving signal. As a main result, we find that at a sufficiently large system-bath coupling strength (i.e., for a friction strength $\alpha>1$ ) the thermal noise plays a constructive role in yielding forced synchronization. This noise-induced synchronization can occur when the driving frequency is larger than the zero-temperature tunneling rate. As an application evidencing the effect, we consider the charge transfer dynamics in molecular complexes.
\end{abstract}

DOI: 10.1103/PhysRevLett.97.210601

PACS numbers: 05.60.Gg, 05.40.-a, 05.45.Xt, 82.20.Gk

The study of the different versions of synchronization appearing in nonlinear classical systems has gained importance over the past decade [1-4]. A special class of problems is provided by noise-induced forced synchronization in driven bistable nonlinear systems $[2,5,6]$. Here a stochastic phase process can be associated with the jumping events between two domains of attraction. The locking of the average frequency of the phase process to that of the external driving and the smallness of the phase-diffusion coefficient in a corresponding interval of noise strengths are the fingerprints of such noise-induced forced synchronization [7-9]. Another manifestation of the rich dynamics of such systems is stochastic resonance (SR) [10], which recently has been generalized to the quantum regime [1113]. Its experimental realization on the level of a nanomechanical quantum memory element is now feasible [14]. Although synchronization and SR are related, the existence of SR does not necessarily imply a (phase) synchronization, as emphasized in Ref. [5]. The extension of noiseinduced synchronization into the realm of quantum physics has not been considered thus far. This latter task presents a challenge which, apart from prominent academic interest, also comprises a great potential for nanoscience with beneficial applications ranging from quantum control to quantum information processing. With this work, we undertake a first step in this direction.

Dissipative quantum tunneling changes radically the physics of classical synchronization. At zero temperature, the system can only tunnel towards its lowest energy state when a biasing dc signal is applied. As the bias periodically changes its sign due to the action of a driving field, tunneling causes the particle to move periodically towards its corresponding lowest energy state, as long as the driving period is much longer than the typical time scale for tunneling. Consequently, one expects that the system may synchronize when driven by a periodic, e.g., rectangular-shaped, signal. By contrast, in the absence of thermal noise, synchronization in overdamped classical bistable systems driven by subthreshold signals fails as no overbarrier transitions occur.

Two interesting questions now emerge: What is the effect of the generally deteriorating thermal quantum noise at finite temperatures on synchronization? How does the time scale of the external driving period impact the quality for (phase) synchronization? Quantum bath fluctuations at finite temperature surely will disturb the above mentioned perfect (phase) synchronization as imposed by the rocking driving field. At the same time, when the driving period becomes shorter than the tunneling time, the zerotemperature synchronization will also be weakened. It might become likely, nevertheless, that finite temperature quantum noise does promote and assist synchronization upon decreasing driving period.

We demonstrate in this Letter that this is indeed the case within the following archetype setup of a dissipative, driven two-level system (TLS) [13,15-17]:

$$
\begin{aligned}
\hat{H}(t)= & \frac{1}{2} \epsilon(t) \hat{\sigma}_{z}+\frac{1}{2} \hbar \Delta \hat{\sigma}_{x}+\frac{1}{2} \hat{\sigma}_{z} \sum_{j} \kappa_{j}\left(\hat{b}_{j}^{\dagger}+\hat{b}_{j}\right) \\
& +\sum_{j} \hbar \omega_{j}\left(\hat{b}_{j}^{\dagger} \hat{b}_{j}+\frac{1}{2}\right) .
\end{aligned}
$$

Herein, the operators $\hat{\sigma}_{z}$ and $\hat{\sigma}_{x}$ denote the standard Pauli operators, $\epsilon(t)$ is a time-dependent energy bias, and $\hbar \Delta$ is the tunnel matrix element. The bath Hamiltonian [last term in Eq. (1)] is expressed in terms of the operators $\hat{b}_{j}^{+}$and $\hat{b}_{j}$ associated to the $j$ th bath normal mode with frequency $\omega_{j}$. The stochastic influence of the quantum thermal bath is captured by an operator random force $\hat{\xi}(t)=$ $\sum_{j} \kappa_{j}\left(\hat{b}_{j}^{\dagger} e^{i \omega_{j} t}+\hat{b}_{j} e^{-i \omega_{j} t}\right)$. It can be characterized by the spectral density $J(\omega)=(\pi / \hbar) \sum_{j} \kappa_{j}^{2} \delta\left(\omega-\omega_{j}\right)$. We assume that $J(\omega)$ acquires the Ohmic form $J(\omega)=$ $2 \pi \hbar \alpha \omega e^{-\omega / \omega_{c}}$, with friction strength $\alpha$ and an exponential cutoff. Because of the Gaussian character of a harmonic bath, the statistical properties of the quantum noise are 
determined solely by its equilibrium autocorrelation function $\langle\hat{\xi}(t) \hat{\xi}(0)\rangle_{T}=(\hbar / \pi) \int_{0}^{\infty} J(\omega)\left[\operatorname{coth}\left(\hbar \omega /\left(2 k_{B} T\right)\right) \times\right.$ $\cos (\omega t)-i \sin \omega t] d \omega$. This driven spin-boson Hamiltonian (1) describes an abundance of applications [18] such as, e.g., the electron transfer (ET) in a molecular dimer in azurin crystals [19]. There the low-frequency molecular vibrations provide the bath, and the timedependent energy bias is given by $\epsilon(t)=r e \mathcal{E}(t)$, where $r$ is the tunneling distance, $e$ denotes the charge transferred, and $\mathcal{E}(t)$ is the time-dependent, applied electric field.

In the incoherent tunneling regime, the populations of the localized states obey a nonstationary, Markovian dynamics. In the presence of a time-dependent bias, this description holds true for Ohmic friction at an arbitrary temperature if the tunnel coupling remains small, i.e., $\Delta \ll$ $\omega_{c}$, and the coupling to the heat bath is sufficiently strong, $\alpha>1 / 2$ [17]. The populations $p_{\beta}(t) \equiv p_{ \pm}(t)=$ $\left(1 \pm\left\langle\hat{\sigma}_{z}(t)\right\rangle_{T}\right) / 2$ obey the balance equations $[13,15,20]$

$$
\dot{p}_{\beta}(t)=W_{-\beta}(t) p_{-\beta}(t)-W_{\beta}(t) p_{\beta}(t),
$$

where the time-dependent relaxation rates $W_{\beta}(t)$ within the golden rule approximation read:

$$
\begin{aligned}
W_{ \pm}(t)= & \frac{1}{2} \Delta^{2} \int_{0}^{\infty} d \tau \exp \left[-Q^{\prime}(\tau)\right] \\
& \times \cos \left[Q^{\prime \prime}(\tau) \mp \frac{1}{\hbar} \int_{t-\tau}^{t} \epsilon\left(t^{\prime}\right) d t^{\prime}\right] .
\end{aligned}
$$

The functions $Q^{\prime}(t)$ and $Q^{\prime \prime}(t)$ denote the real and imaginary parts of the dissipation kernel

$$
Q(t)=\frac{i \lambda t}{\hbar}+\frac{1}{\hbar^{2}} \int_{0}^{t} d t_{1} \int_{0}^{t_{1}} d t_{2}\left\langle\hat{\xi}\left(t_{2}\right) \hat{\xi}(0)\right\rangle_{T},
$$

wherein $\lambda=\int_{0}^{\infty} d \omega J(\omega) /(\pi \omega)$ is the bath reorganization energy [20]. With $\lambda=2 \alpha \hbar \omega_{c}$, one finds that

$$
\begin{gathered}
Q^{\prime}(t)=2 \alpha \ln \left\{\sqrt{1+\omega_{c}^{2} t^{2}} \frac{\Gamma^{2}(1+\kappa)}{\left|\Gamma\left(1+\kappa+i \omega_{T} t\right)\right|^{2}}\right\}, \\
Q^{\prime \prime}(t)=2 \alpha \arctan \left(\omega_{c} t\right) .
\end{gathered}
$$

In Eq. (5), $\Gamma(z)$ denotes the Gamma function, $\omega_{T}=$ $k_{B} T / \hbar$, and $\kappa=\omega_{T} / \omega_{c}$. The most relevant regime for our purpose corresponds to $\hbar \Delta \ll \hbar \omega_{c}, \lambda$ and $\alpha>1$. Note that in the limit of an adiabatic driving, varying on a time scale $\mathcal{T}$ obeying $\omega_{c} \mathcal{T}, \alpha \omega_{T} \mathcal{T} \gg 1$, the timedependent transition rates $W_{ \pm}(t)$ follow the instantaneous value of the bias $\epsilon(t)$. In this limit, which in the following is assumed throughout, the relaxation rates $W_{ \pm}(t)$ obey the Boltzmann relation $W_{-}(t)=\exp \left(-\epsilon(t) /\left(k_{B} T\right)\right) W_{+}(t)$. Furthermore, in the high-temperature limit $k_{B} T \gg \hbar \omega_{c}$ and the quasistatic noise approximation, i.e., $\langle\hat{\xi}(t) \hat{\xi}(0)\rangle_{T} \approx$ $\left\langle[\hat{\xi}(0)]^{2}\right\rangle_{T}$ in Eq. (4), Eq. (3) reduces to a generalized Marcus-Levich-Dogonadze form, reading $W_{ \pm}(t)=$ $(\pi / 2) \hbar \Delta^{2} / \sqrt{4 \pi \lambda k_{B} T} \exp \left[-( \pm \epsilon(t)-\lambda)^{2} /\left(4 \lambda k_{B} T\right)\right]$. For $k_{B} T \leq \hbar \omega_{c}$, explicit analytical expressions for the rates are generally not available, except for $T=0$ [17].
Typically, those must be determined numerically from Eq. (3). The quantum rate $W_{+}$displays a non-Arrhenius dependence on temperature, being rather a power law that typifies the low-temperature limit.

Using the Marcus-Levich-Dogonadze formula for an undriven molecular system yields an estimate for the relevant parameter values. For ET occurring in an azurin dimer, the values are $\lambda=0.25 \mathrm{eV}$ and $\hbar \Delta=5 \times$ $10^{-6} \mathrm{eV}$ [19]. In molecular systems, the cutoff frequency of low-frequency molecular vibrations ranges between $\hbar \omega_{c}=5$ and $20 \mathrm{meV}$. Choosing $\hbar \omega_{c}=12.5 \mathrm{meV}$ yields $\alpha=10$, being consistent with an incoherent tunneling dynamics.

It is worth noting that the present incoherent limit for the tunneling dynamics of a driven, dissipative TLS allows for an effective quasiclassical interpretation in terms of a classical, time-inhomogeneous random telegraph process. Its transition rates, however, are governed by a manifest quantum dynamics, as detailed with Eq. (3). Our setup thus mimics the quantum analogue of a classical (phase) synchronization behavior elaborated in Refs. [7-9]. A thought-experimental setup is that of a particle tunneling between two localized states and subjected to an external periodic rectangular field with amplitude $\mathcal{E}_{0}$ and period $\mathcal{T}$ (frequency $\Omega=2 \pi / \mathcal{T}$ ). One then counts the number of jumps $n(t)$ within a time window $\left[t_{0}, t\right)$. We introduce the random phase $\phi\left(t, t_{0}\right)=\pi n(t)$, which increases by $\pi$ at each switching event (two subsequent switches correspond to a $2 \pi$ cycle of random duration) and define the average frequency and diffusion coefficients associated to the stochastic phase process as $\bar{\Omega}_{\mathrm{ph}}:=\lim _{t \rightarrow \infty}\left\langle\phi\left(t, t_{0}\right)\right\rangle /\left(t-t_{0}\right)$ and $2 \bar{D}_{\mathrm{ph}}:=\lim _{t \rightarrow \infty}\left[\left\langle\phi^{2}\left(t, t_{0}\right)\right\rangle-\left\langle\phi\left(t, t_{0}\right)\right\rangle^{2}\right] /\left(t-t_{0}\right)$, respectively. To evaluate these quantities, one considers the joint probability $P_{\beta, n}(t)$ to be in the state $\beta$ at time $t$ and to have undergone $n$ jumps within the time interval $\left[t_{0}, t\right)$. These probabilities can be obtained by integrating the multitime probability densities of the corresponding stochastic trajectories [21]. They satisfy the normalization condition $\sum_{n=0}^{\infty} \sum_{\beta= \pm} P_{\beta, n}(t)=1$ and obey for $n \geq 1$ the master equation:

$$
\dot{P}_{\beta, n}(t)=W_{-\beta}(t) P_{-\beta, n-1}(t)-W_{\beta}(t) P_{\beta, n}(t),
$$

whereas $\dot{P}_{\beta, 0}(t)=-W_{\beta}(t) P_{\beta, 0}(t)$. The populations of the states can be obtained as $p_{\beta}(t)=\sum_{n=0}^{\infty} P_{\beta, n}(t)$, and the probability $P_{n}(t)$ for $n$ jumps is $P_{n}(t)=P_{+, n}(t)+P_{-, n}(t)$. Given $P_{n}(t)$, the moments are $\left\langle n^{k}(t)\right\rangle:=\sum_{n=0}^{\infty} n^{k} P_{n}(t)$ $(k=1,2, \ldots)$. Deriving explicit analytical expressions, however, presents a nontrivial task. Fortunately, the first two moments present exceptions to this rule. In particular, from Eqs. (2) and (7), it follows that the (phase) frequency $\Omega_{\mathrm{ph}}(t):=\pi(d / d t)\langle n(t)\rangle$ can be expressed as

$$
\Omega_{\mathrm{ph}}(t)=\pi\left[W_{+}(t) p_{+}(t)+W_{-}(t) p_{-}(t)\right] .
$$

For the averaged phase, we find $\left\langle\phi\left(t, t_{0}\right)\right\rangle=\int_{t_{0}}^{t} \Omega_{\mathrm{ph}}\left(t^{\prime}\right) d t^{\prime}$. To obtain $\bar{\Omega}_{\mathrm{ph}}$, we take the limit $\bar{\Omega}_{\mathrm{ph}}=$ 
$\lim _{t \rightarrow \infty}\left\langle\phi\left(t, t_{0}\right)\right\rangle /\left(t-t_{0}\right)$. With a periodic driving the asymptotic solutions for both, $p_{\beta}(t)$ and $\Omega_{\mathrm{ph}}(t)$ are periodic functions in time with the period $\mathcal{T}$. Thus, $\bar{\Omega}_{\mathrm{ph}}=$ $(1 / \mathcal{T}) \int_{0}^{\mathcal{T}} d t \Omega_{\mathrm{ph}}^{(\infty)}(t)$, where $\Omega_{\mathrm{ph}}^{(\infty)}(t)$ is given by Eq. (8) with $p_{\beta}(t)$ replaced by the asymptotic solution $p_{\beta}^{(\infty)}(t)$ of the master equation (2), which formally is obtained by letting $t_{0} \rightarrow-\infty$.

For $D_{\mathrm{ph}}(t):=\left(\pi^{2} / 2\right)(d / d t)\left[\left\langle n^{2}(t)\right\rangle-\langle n(t)\rangle^{2}\right]$, a cumbersome evaluation following Ref. [8] yields

$$
\begin{aligned}
2 D_{\mathrm{ph}}(t)= & \pi \Omega_{\mathrm{ph}}(t)-2 \pi^{2} \delta W(t) \\
& \times \sum_{\beta= \pm} \beta \int_{t_{0}}^{t} d t^{\prime} W_{\beta}\left(t^{\prime}\right) p_{\beta}^{2}\left(t^{\prime}\right) \exp \left[-\int_{t^{\prime}}^{t} W(\tau) d \tau\right]
\end{aligned}
$$

where $\delta W(t)=W_{+}(t)-W_{-}(t) \quad$ and $\quad W(t)=W_{+}(t)+$ $W_{-}(t)$. The evaluation of the mean phase-diffusion coefficient $\bar{D}_{\mathrm{ph}}$ proceeds similarly to $\bar{\Omega}_{\mathrm{ph}}$. For the considered case of a rectangular-shaped driving of strength $\epsilon_{0}$, i.e., $\epsilon(t)= \pm \epsilon_{0}$, our results are

$$
\bar{\Omega}_{\mathrm{ph}}=\frac{\pi W}{2}\left\{1-\delta p_{\mathrm{eq}}^{2}\left[1-\frac{4 \tanh (W \mathcal{T} / 4)}{W \mathcal{T}}\right]\right\}
$$

and

$$
\begin{aligned}
2 \bar{D}_{\mathrm{ph}}= & \pi \bar{\Omega}_{\mathrm{ph}}-\frac{2 \pi^{2}}{\mathcal{T}} \delta p_{\mathrm{eq}}^{4} \tanh ^{3}(W \mathcal{T} / 4) \\
& -\frac{\pi^{2}}{2 \mathcal{T}} \delta p_{\mathrm{eq}}^{2}\left(1-\delta p_{\mathrm{eq}}^{2}\right)\{12 \tanh (W \mathcal{T} / 4) \\
& \left.-W \mathcal{T}\left[1+2 \operatorname{sech}^{2}(W \mathcal{T} / 4)\right]\right\},
\end{aligned}
$$

where $W$ denotes the sum of the forward and backward rates in Eq. (3) for a fixed value of the field $\mathcal{E}_{0}$, i.e., for $\epsilon(t)=\epsilon_{0}=e r \mathcal{E}_{0}$, and $\delta p_{\text {eq }}=\tanh \left(\epsilon_{0} /\left(2 k_{B} T\right)\right)$ is the absolute value of the difference of the equilibrium populations. The inverse Fano factor of the counting process $R:=\pi \bar{\Omega}_{\mathrm{ph}} /\left(2 \bar{D}_{\mathrm{ph}}\right)$ provides a reliable quality measure of forced synchronization $[5,6]$.

The quantum features of synchronization are rooted in the quantum rate expressions entering Eqs. (10) and (11). Using the analogy with quantum stochastic resonance in symmetric quantum systems [11], one would then expect that no thermal noise-assisted synchronization occurs for $\alpha \leq 1$. Indeed, we could not identify noise-assisted synchronization within this parameter regime. On the same token, we expect synchronization to emerge for friction strength $\alpha>1$ [12].

Indeed, for $\alpha>1$, the synchronization scenario sensitively depends on the value of the driving frequency. As discussed above, for driving frequencies much smaller than the tunneling rate at $T=0$, i.e., $\Omega \ll W_{T=0}=$ $\pi \Delta^{2}\left[2 \omega_{c} \Gamma(2 \alpha)\right]^{-1}\left[\epsilon_{0} /\left(\hbar \omega_{c}\right)\right]^{2 \alpha-1} \exp \left[-\epsilon_{0} /\left(\hbar \omega_{c}\right)\right], \quad \bar{\Omega}_{\mathrm{ph}}$ matches the external frequency for sufficiently low temperatures. This is depicted in Fig. 1(a), where the behavior of $\bar{D}_{\mathrm{ph}}$ is presented as well. Notice that quantum synchronization at sufficiently low temperature is impressively achieved, as manifested by the large values of the $R$ factor; see Fig. 1(c). As the temperature increases above a certain threshold value, however, the $R$ factor diminishes and the quality of synchronization deteriorates.

Upon increasing the driving frequency further, beyond the regime $\Omega \ll W_{T=0}$, we enter the regime of thermally induced phase synchronization in the quantum regime, as depicted in Fig. 1(b). We detect a whole temperature interval where the external frequency $\Omega$ and $\bar{\Omega}_{\mathrm{ph}}$ ideally coincide. Moreover, the accompanying phase-diffusion coefficient exhibits a distinct, cusplike minimum. These results convincingly illustrate the constructive role of quantum thermal noise for quantum synchronization. Notice that with increasing driving frequency the quality factor associated to the synchronization effect decreases; see Fig. 1(c). Furthermore, numerics indicate that the temperature range for frequency locking shrinks. The quality factor $R$ displays a cusplike feature for large $R_{\max }$ values at maximum, which is similar in spirit to the one revealed recently by Park and Lai [22] within classical, forced stochastic synchronization [23].

Increasing the driving frequency further, the tunneling rate is too slow in order to follow the external driving; consequently, quantum synchronization is lost. This be-
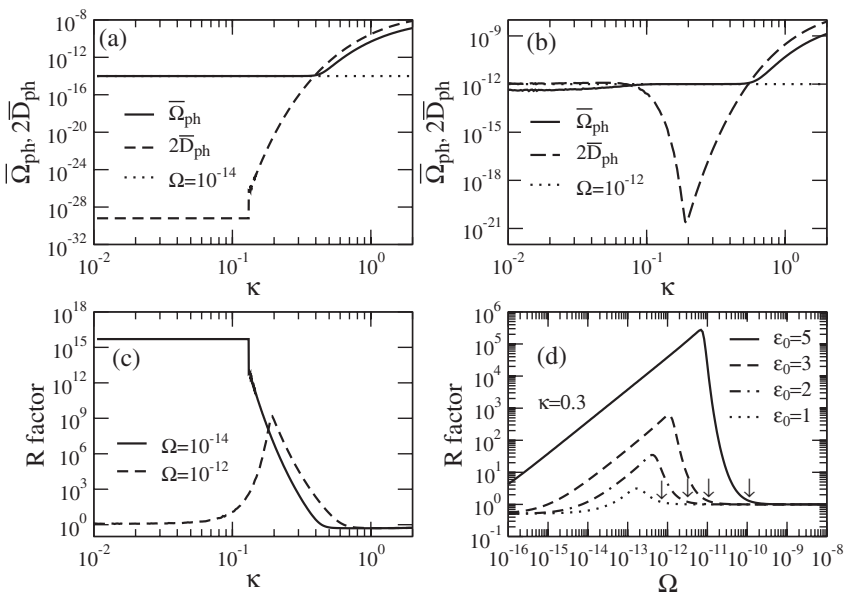

FIG. 1. (a)-(c) Stochastic frequency of quantum tunneling jumps $\bar{\Omega}_{\mathrm{ph}}$, phase-diffusion coefficient $\bar{D}_{\mathrm{ph}}$, and the synchronization quality factor $R$ vs the scaled temperature $\kappa=k_{B} T / \hbar \omega_{c}$ for two values of the angular driving frequency $\Omega$. (d) Quality factor $R$ vs driving frequency $\Omega$ at the fixed temperature $\kappa=0.3$ and for four driving strengths $\epsilon_{0}=1,2,3$, and 5. The arrows indicate the resonant driving frequencies which are expected from matching the inverse tunneling rate $1 / W_{+}\left(\epsilon_{0}\right)$ with the driving half-period, i.e., $\pi W_{+}=\Omega_{\mathrm{SR}}$. The used parameter values are $\alpha=10, \Delta=4 \times 10^{-4}$, and $\epsilon_{0}=5$ (a)-(c). The frequencies are scaled with $\omega_{c}$ and the energies with $\hbar \omega_{c}$. For $\hbar \omega_{c}=12.5 \mathrm{meV}, \quad \omega_{c} \approx 1.9 \times 10^{13} 1 / \mathrm{sec}, \quad$ and $r=14.9 \AA$ (chosen to match the charge transfer in molecules from Ref. [19]), one obtains a field strength of $\mathcal{E}_{0} \approx 4.19 \times$ $10^{4} \mathrm{~V} / \mathrm{cm}$. The value $\kappa=1$ corresponds to $145 \mathrm{~K}$. 
havior is depicted in Fig. 1(d) for different driving strengths at a fixed temperature. The range of synchronization corresponds to the linearly rising part of the $R(\Omega)$ dependence. The optimum of synchronization occurs at frequencies $\Omega_{\max }$ lying below the corresponding frequencies $\Omega_{\mathrm{SR}}$ that optimize SR. This is so because SR requires a matching of the mean tunneling time with the driving halfperiod $\mathcal{T} / 2$, whereas for phase synchronization the particle must tunnel before the bias alternates. Very probable is, however, that during $\mathcal{T} / 2$ the tunneling is not yet completed. This leads to desynchronization at $\Omega_{\mathrm{SR}}$. Moreover, synchronization is also lost in Fig. 1(d) for a driving strength $\epsilon_{0}=1$ (no frequency locking is present) although $R(\Omega)$ displays a resonantlike behavior, a feature which can be correlated with SR [9].

In conclusion, we have unravelled the existence of a quantum stochastic synchronization in a driven spin-boson system undergoing tunneling transitions between two states. We exemplified the phenomenon for nonadiabatic charge transfer in molecular complexes. The estimates of parameter values for an experimental test of our theoretical predictions are (cf. Fig. 1 caption) driving frequencies $\Omega \sim 10^{-1}-10^{3} \mathrm{~s}^{-1}$, electrical field strength $\mathcal{E}_{0} \sim$ $10^{4}-10^{5} \mathrm{~V} / \mathrm{cm}$, and temperatures $T \sim 20-100 \mathrm{~K}$, which are all readily achieved in the laboratory. The main quantum features of the discussed synchronization phenomenon are robust, and they are not critically dependent on the details of the underlying dissipation mechanism. This is so because the phenomenon of quantum synchronization is primarily based on the existence of a low-temperature limit of the tunneling rates. We thus expect our very general results to be of constructive use in different contexts such as, e.g., for optimizing the noisy dynamics of nanomechanical systems in the quantum regime [14]. The authors are confident that these riveting findings for quantum synchronization will spur experimental interests for diverse systems that involve a controllable quantum tunneling between distinct quantum states.

J.C.-P. and M.M. acknowledge the support of the Ministerio de Educación y Ciencia of Spain (No. FIS2005-02884) and the Junta de Andalucía. I. G. and P.H. acknowledge support by the DFG through No. SFB 486 and the ESF program "Stochdyn."

[1] A. Pikovsky, M. Rosenblum, and J. Kurths, Synchronization: A Universal Concept in Nonlinear Science (Cambridge University Press, Cambridge, England, 2001).

[2] V. Anishchenko, A. Neiman, A. Astakhov, T. Vadiavasova, and L. Schimansky-Geier, Chaotic and Stochastic Processes in Dynamic Systems (Springer, Berlin, 2002).

[3] S. Boccaletti et al., Phys. Rep. 366, 1 (2002).

[4] S. Strogatz, Sync: The Emerging Science of Spontaneous Order (Hyperion, New York, 2003).
[5] J. A. Freund, L. Schimansky-Geier, and P. Hänggi, Chaos 13, 225 (2003).

[6] B. Lindner, J. Garcia-Ojalvo, A. Neiman, and L. Schimansky-Geier, Phys. Rep. 392, 321 (2004).

[7] J. A. Freund, A. B. Neiman, and L. Schimansky-Geier, Europhys. Lett. 50, 8 (2000).

[8] J. Casado-Pascual et al., Phys. Rev. E 71, 011101 (2005); J. Casado-Pascual, J. Gómez-Ordóñez, and M. Morillo, Physica (Amsterdam) 351A, 117 (2005).

[9] P. Talkner, Physica (Amsterdam) 325A, 124 (2003); P. Talkner et al., New J. Phys. 7, 14 (2005).

[10] L. Gammaitoni et al., Rev. Mod. Phys. 70, 223 (1998); P. Hänggi, Chem. Phys. Chem. 3, 285 (2002); J. CasadoPascual, J. Gómez-Ordóñez, and M. Morillo, Chaos 15, 026115 (2005).

[11] R. Löfstedt and S. N. Coppersmith, Phys. Rev. Lett. 72, 1947 (1994); Phys. Rev. E 49, 4821 (1994); M. Grifoni and P. Hänggi, Phys. Rev. Lett. 76, 1611 (1996); M. Grifoni and P. Hänggi, Phys. Rev. E 54, 1390 (1996); M. Grifoni, L. Hartmann, S. Berchtold, and P. Hänggi, Phys. Rev. E 53, 5890 (1996); 56, 6213(E) (1997); D. E. Makarov and N. Makri, Phys. Rev. B 52, R2257 (1995); K. Dong and N. Makri, Phys. Rev. A 70, 042101 (2004).

[12] I. Goychuk and P. Hänggi, Phys. Rev. E 59, 5137 (1999); I. Goychuk and P. Hänggi, New J. Phys. 1, 14 (1999).

[13] M. Grifoni and P. Hänggi, Phys. Rep. 304, 229 (1998).

[14] R. L. Badzey and P. Mohanty, Nature (London) 437, 995 (2005).

[15] I. Goychuk and P. Hänggi, Adv. Phys. 54, 525 (2005).

[16] A. J. Leggett et al., Rev. Mod. Phys. 59, 1 (1987).

[17] U. Weiss, Quantum Dissipative Systems (World Scientific, Singapore, 1999), 2nd ed.

[18] H. Grabert and A. Nitzan, Chem. Phys. 296, 101 (2004).

[19] K. V. Mikkelsen, L. K. Skov, H. Nar, and O. Farver, Proc. Natl. Acad. Sci. U.S.A. 90, 5443 (1993).

[20] I. A. Goychuk, E. G. Petrov, and V. May, Phys. Rev. E 52, 2392 (1995); I. A. Goychuk, E. G. Petrov, and V. May, Chem. Phys. Lett. 253, 428 (1996); Y. Dakhnovskii, Phys. Rev. B 49, 4649 (1994); R. I. Cukier and M. Morillo, J. Chem. Phys. 91, 857 (1989); M. Morillo and R. I. Cukier, Phys. Rev. B 54, 13962 (1996).

[21] I. Goychuk and P. Hänggi, Phys. Rev. E 61, 4272 (2000).

[22] K. Park and Y.-C. Lai, Europhys. Lett. 70, 432 (2005).

[23] This feature is lost with the smaller peak value at $R=$ $R_{\max }$ and increasing driving frequency $\Omega$. The dependence of $R_{\max }$ on the friction strength $\alpha$ at fixed driving frequency and amplitude is elucidative as well: For $\Omega=$ $10^{-10}, \epsilon_{0}=5$, and $\Delta=4 \times 10^{-4}$ (in units of $\omega_{c}$ and $\hbar \omega_{c}$, respectively) and within $6.4 \leq \alpha \leq 15$, a numerical study reveals that $R_{\max } \approx \exp \left[a\left(\alpha-\alpha_{\mathrm{th}}\right)^{-\gamma}\right]$, with $a \approx 44.2$, $\alpha_{\text {th }} \approx 5.33$, and $\gamma \approx 1.35$. For $\alpha$ approaching $\alpha_{\text {th }}, R_{\max }$ rapidly increases. A similar hypersensitive dependence is observed in the context of mutual synchronization [24]. Moreover, the dependence of $R_{\max }$ vs $\epsilon_{0}$ grows faster than exponential.

[24] Y.-C. Lai, M. G. Frei, and I. Osorio, Phys. Rev. E 73, 026214 (2006). 